\title{
COMMISSIONING RESULTS OF THE NARROW-BAND BEAM POSITION MONITOR SYSTEM UPGRADE IN THE APS STORAGE RING ${ }^{\circ}$
}

\author{
O. Singh, C. Doose, J. Carwardine, G. Decker, F. Lenkszus and R. Merl \\ Advanced Photon Source, Argonne, IL 60439 USA
}

\section{Abstract}

When using a low emittance storage ring as a high brightness synchrotron radiation source, it is critical to maintain a very high degree of orbit stability, both for the short term and for the duration of an operational fill. A fill-to-fill reproducibility is an additional important requirement. Recent developments in orbit correction algorithms have provided tools that are capable of achieving a high degree of orbit stability. However, the performance of these feedback systems can be severely limited if there are errors in the beam position monitors (BPMs). The present orbit measurement and correction system at the APS storage ring utilizes 360 broad-bandtype BPMs that provide turn-by-turn diagnostics and an ultra-stable orbit: $<1.8$ micron $\mathrm{rms}$ vertically and 4.5 microns rms horizontally in a frequency band of 0.017 to $30 \mathrm{~Hz}$. The effects of beam intensity and bunch pattern dependency on these BPMs have been significantly reduced by employing "offset compensation" correction. Recently, 40 narrow-band switching-type BPMs have been installed in the APS storage ring, two in each of 20 operational insertion device straight sections, bringing the total number of beam position monitors to 400 . The use of narrow-band BPM electronics is expected to reduce sensitivity to beam intensity, bunch pattern dependence, and long-term drift. These beam position monitors are used for orbit correction/feedback and machine protection interlocks for the insertion device beamlines. The commissioning results and overall performance for orbit stability are provided.

\section{INTRODUCTION}

The third-generation synchrotron light sources, such as the Advanced Photon Source (APS) storage ring, must meet very tight orbit stability requirements needed for low-emittance charged particle beams. These requirements get even tighter as the beam size reduces further. The orbit stability work at APS is at the forefront in many ways; here, we will discuss results of recently commissioned narrow-band switching-type beam position monitors (NBBPMs), connected to the insertion device chambers.

This type of BPM, first developed in the late 1980s [1], was followed by several design improvements $[2,3]$, particularly a significant increase in the input dynamic range. The bulky chassis-type package has been reduced to a single height Euro-type module with several practical built-in features. Such a commercially available. Forty of these units have been integrated together with the existing 360 broad-bandtype or monopulse beam position monitors (MPBPM). Front-end upgrade work on the MPBPM system is also in progress, which will enhance the global orbit stability performance [4].

Two orbit correction systems - "fast" [5] and "slow" [6] - that correct the orbit up to about $50 \mathrm{~Hz}$ have been employed at the APS storage ring. Both systems make orbit correction only for the long spatial wavelength motions, taking great statistical advantage of a large number of BPMs, thus not responding to local artificial effects that may be exhibited by individual BPMs. The "offset compensations," based upon "scrape down" fitted data [6], are made to the raw BPM data. This reduces a large number of systematic errors, such as intensity/bunch pattern dependency and thermal effect in the data, presented to the orbit correction algorithms.

The bench data for NBBPMs show that the beam intensity dependence is less than 2 microns in the upper $40 \mathrm{~dB}$ of the power range, but it is challenging to make similar claims in the storage ring. Uncertainty in the orbit itself and the thermally induced chamber motion are some of the culprits that contaminate the measurements. The high performance $x$-ray-type beam position monitors (XBPMs) [7] have been routinely used as a reliable reference, but only for the bending magnet (BM) sources. However, recent work done by modifying the lattice [8] for one insertion device may hold the key to future use of XBPMs as a reference for ID sources as well.

\section{INSTALLATION/COMMISSIONING}

There are ten Eurocrates installed around half the ring, each housing NBBPM modules for two sectors. The NBBPM output signals are sent to a digitizing beam position limit detector (DBPLD) for machine protection [9]. The response time requirement of 350 microseconds for a beam deflection of $+/-1 \mathrm{~mm}$ is easily met. A 300 $\mathrm{Hz}$ anti-aliasing filter module is used to provide input to a 16-bit orbit measurement digitizer that samples at the orbit feedback rate of $1.6 \mathrm{kHz}$. This sampled data is fed to the real-time feedback system and to an averager that then passes data to the "slow" correction system. The NBBPM calibrations for the $8-\mathrm{mm}$ chamber are $3 \mathrm{~V} / \mathrm{mm}$ and $5 \mathrm{~V} / \mathrm{mm}$ for vertical and horizontal planes, respectively. 


\section{DISCLAIMER}

This report was prepared as an account of work sponsored by an agency of the United States Government. Neither the United States Government nor any agency thereof, nor any of their employees, make any warranty, express or implied, or assumes any legal liability or responsibility for the accuracy, completeness, or usefulness of any information, apparatus, product, or process disclosed, or represents that its use would not infringe privately owned rights. Reference herein to any specific commercial product, process, or service by trade name, trademark, manufacturer, or otherwise does not necessarily constitute or imply its endorsement, recommendation, or favoring by the United States Government or any agency thereof. The views and opinions of authors expressed herein do not necessarily state or reflect those of the United States Government or any agency thereof. 


\section{DISCLAIMER}

Portions of this document may be illegible in electronic image products. Images are produced from the best available original document. 
For a long undulator $\left(\sigma_{Y} \ll \sigma_{x}\right)$ and a centered beam waist $(Z=0)$, Eq. (3) predicts that the measured effective size is smaller than the actual beam, due to the high collimation of the undulator beams. While the electrons' angular distribution is independent of their positions at the beam waist, the farther off-axis the electrons, the fewer photons they generate will pass through the pinhole. This results in a systematic error of a smaller measured effective source size, as pointed out previously $[2,3,5]$. This expression, based on simple geometrical optics models, applies only to the horizontal direction, where the beam size is much larger than the width of the camera point-spread-function (PSF).

\subsection{Horizontal Beam Emittance}

The expressions for effective divergence and beam size both show strong dependence on $\beta$ functions, as well as the location of the beam waist, thus making them susceptible to magnetic lattice fluctuations. The product of these two numbers, however, is less dependent on them,

$$
\begin{aligned}
& \varepsilon_{x, f f}=\sigma_{x, f f} \sigma_{x^{\prime} \in f f} \\
& =\varepsilon \sqrt{\frac{\beta_{0}{ }^{2}+\left(S_{m}-Z\right)^{2}+S_{m}{ }^{2} \frac{\sigma_{m}{ }^{2}}{\sigma_{x}^{2}}}{\beta_{0}{ }^{2}+(S-Z)^{2}+S^{2} \frac{\sigma_{r}^{2}}{\sigma_{x}^{2}}} \cdot\left(1+\frac{\beta \sigma_{r}^{2}}{\beta_{0} \sigma_{x^{2}}{ }^{2}}\right)},
\end{aligned}
$$

where $\beta_{0}$ is the $\beta$ function value at the beam waist.

(1) When the pinhole camera and the divergence measurement are using $x$-rays of the same energy, we may choose the location of monochromator and $x$-ray slits to be at the same distance from the undulator, $S_{m}=S$. Equation (4) can be further simplified to

$$
\varepsilon \cong \sigma_{x, e f f} \sigma_{x \rightarrow f f},
$$

with a small correction term (less than $1 \%$ for the APS diagnostics undulator, $\sigma_{\gamma} \cong 2.6 \mu \mathrm{rad}$ and $\sigma_{\mathrm{x}^{\prime}} \cong 20 \mu \mathrm{rad}$ ).

(2) When the pinhole camera is operating with a broad-band x-ray beam, $\sigma_{r}$ increases somewhat, but the correction to Eq. (5) is still expected to be small.

\subsection{Fresnel Diffraction Broadening in y-Direction}

The diffraction broadening of the pinhole image has been discussed by several authors [2,3], based on the hybrid model in which the geometric shadow of the pinhole and the Fraunhofer diffraction pattern were both approximated by Gaussian function, and the total broadening was assumed to be their convolution. Borland [5] appeared to be the first to point out the inadequacy of Fraunhofer diffraction and briefly discussed the effect of Fresnel diffraction. We have performed a detailed analysis of the PSF based on Fresnel diffraction. The PSF for monochromatic radiation for a pinhole aperture (width $=d$ ) can be written as the square of a Fresnel integral. It can be represented by an integral to allow analytical convolution with a Gaussian source function and a Gaussian spectrum function (polychromatic PSF). The result can then be compared directly with experimentally measured profiles.

\section{EXPERIMENT}

The experiment was performed at the diagnostics beam line of the Advanced Photon Source. The set up is shown in Figure 1 with relevant parameters given in Table 1.

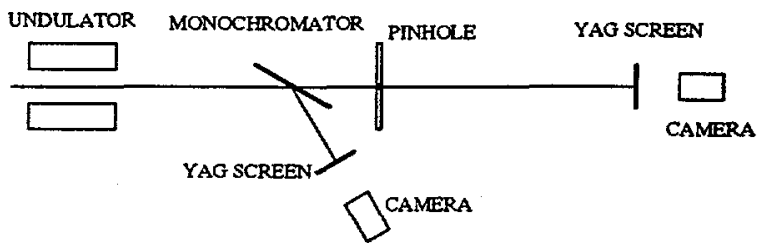

Figure 1: APS diagnostics undulator beamline

Table 1: Parameters for the APS undulator experiment

\begin{tabular}{|l|c|}
\hline Undulator, period length, $\lambda$ & $18 \mathrm{~mm}$ \\
\hline Undulator length, $L$ & $3.56 \mathrm{~m}$ \\
\hline Undulator, fundamental photon energy & $25.9 \mathrm{keV}$ \\
\hline Monochromator to undulator distance, $S$ & $27.47 \mathrm{~m}$ \\
\hline Monochromator crystal & $\mathrm{Si}(400)$ \\
\hline Monochromator crystal thickness & $0.3 \mathrm{~mm}$ \\
\hline Pinhole to undulator distance, $S$ & $28.56 \mathrm{~m}$ \\
\hline Pinhole to X-ray camera distance, $S^{\prime}$ & $9.13 \mathrm{~m}$ \\
\hline
\end{tabular}

\subsection{Divergence Measurement}

The Bragg reflection of a $300-\mu \mathrm{m} \quad \mathrm{Si}(400)$ monochromator crystal was used for the divergence measurement. The crystal angle is chosen to be slightly lower than that for the resonance $\left(10.13^{\circ}\right)$. Integrated intensity profiles were obtained from digitized video images and fitted to Gaussian functions at video frequency $(30 \mathrm{~Hz})$. The data are logged at one-minute interval during user runs. Figure 2 shows such a $\log$ in a 12-hour run during December 1998. At high current in the fill, the horizontal beam size and divergence were high due to a subtle transverse instability [8]. As current decays, the divergences settle to values independent of the current,

$$
\sigma_{x^{\prime}, \text { eff }}=22 . \varepsilon \pm 1(\mu \mathrm{rad}) \text {, and } \sigma_{y^{\prime}, \text { eff }}=3.2 \pm 0.2(\mu \mathrm{rad}) \text {, }
$$

after correction for resolution ( $3 \mu \mathrm{rad})$.

\subsection{Source Size from Pinhole Camera}

The pinhole camera operates with a broad-band x-ray spectrum. The upstream $300-\mu \mathrm{m}$ monochromator crystal at $10^{\circ}$ grazing incidence angle is effectively a $1.73-\mathrm{mm}$ thick silicon filter, which only allows photons above $15 \mathrm{keV}$ to pass. The calculated angular distribution of the transmitted photons fits well to a Gaussian function $\left(\sigma_{r}=20.3 \mu \mathrm{rad}\right)$. We also found that the measured horizontal intensity profile from the pinhole camera has 
a nearly perfect Gaussian shape, giving an effective beam size $\sigma_{\mathrm{x}, \text { eff }}=360(\mu \mathrm{m})$. Correction for instrument resolution is insignificant in this case.
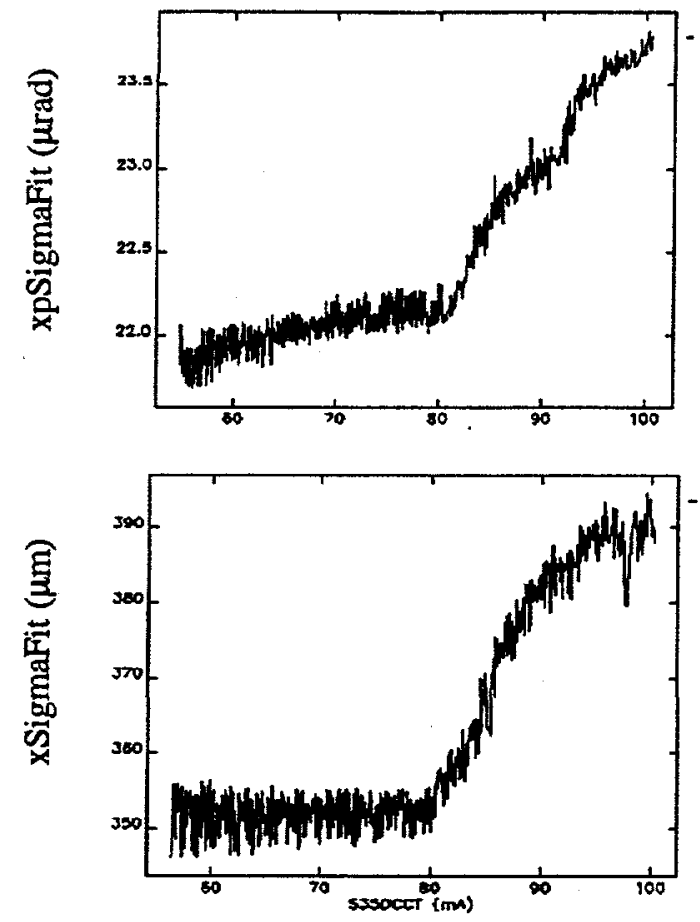

Figure 2: Beam data logged at 1-minute intervals. Upper panel: horizontal beam divergence ( $\mu \mathrm{rad})$; Lower panel: horizontal beam size $(\mu \mathrm{m})$.

\subsection{Horizontal Beam Emittance}

Multiplying the effective beam divergence and size, we obtain the horizontal emittance $\varepsilon_{\mathrm{x}}=7.9 \pm 0.5$ (nm rad). This agrees well with the measurement from the bending magnet sources.

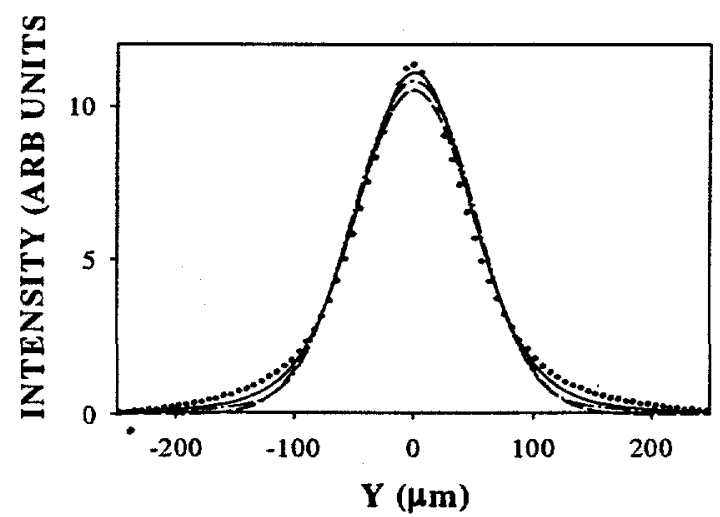

Figure 3: Integrated intensity profile in the vertical direction. (Dots) measured, (dashed line) Gaussian fit, (dash-dot) hybrid model fit with $\sigma_{\mathrm{x}}=24 \mu \mathrm{m}$, and (solid line) Fresnel diffraction fit with $\sigma_{x}=34 \mu \mathrm{m}$.

\subsection{Fresnel Diffraction in the Vertical Direction}

Figure 3 shows the vertical intensity profile obtained from the pinhole image. Attempts to fit the profile with either a Gaussian function or a hybrid profile function failed due to the pronounced center peak and sidelobes. The polychromatic Fresnel model appears to improve the fit with an effective beam size of $\sigma_{y, \text { eff }}=34 \pm 7(\mu \mathrm{m})$. The vertical emittance is thus $\varepsilon_{y}=0.11$ ( $\mathrm{nm} \mathrm{rad}$ ). This represents a vertical coupling of $1.4 \%$, larger than that deduced from the measured beam size at the bending magnet source and measured beta functions.

\section{SUMMARY}

We reach the following conclusions from this work.

(1) By combining a thin monochromator crystal and a pinhole camera with a suitable undulator source, we have demonstrated experimentally that both electron beam divergence and size can be measured simultaneously. The current rate of measurement is limited by the speed of the camera/digitizer at about 30 $\mathrm{Hz}$.

(2) While the lattice function and the properties of the undulator radiation can significantly affect either measured effective beam divergence or size, their product remains a good measure of emittance, robust against fluctuations of lattice $\alpha$ - and $\beta$-functions.

(3) We have presented experimental evidence showing that the Fresnel diffraction is valid and important in understanding $\mathrm{X}$-ray pinhole camera data. Its introduction also pushes the fundamental resolution limit below that of the current, hybrid model by $\sim 30 \%$.

We wish to acknowledge G. Goeppner, I.-C. Sheng, E. Rotela, and S. Sharma for their help in beamline construction; M. Borland and L. Emery for help with the storage ring experiment; and G. Decker and J. Galayda for their continued support for the project.

\section{REFERENCES}

[1] A. Hofmann, "Beam Diagnostics and Applications," AIP Conf. Proc. 451 (3) 1998.

[2] P. Elleaume, C. Fortgang, C. Penel, E. Tarazona, "Measuring Beam Sizes and Ultra-Small Electron Emittances Using an X-Ray Pinhole Camera," J. Synch. Rad. 2, (209) 1995.

[3] C. Zai et al., "Beam Size Measurement of the Stored Electron Beam at the APS Storage Ring Using Pinhole Optics," Rev. Sci. Instrum 67, (3368) 1996.

[4] B. X. Yang and A. H. Lumpkin, "Particle-Beam Profiling Techniques on the APS Storage Ring," AIP Proc. 390 (491) 1997.

[5] M. Borland, "A Method for Calculating emittance from Undulator Images," SSRL ACD-NOTES 60, 1989.

[6] C. Zai et al., "Phase-space Measurement of Stored Electron Beam at the Cornell Electron Storage Ring Using a Combination of Slit Array and CCD Detector," Rev. Sci. Instrum 66, (1859), 1995.

[7] K-J. Kim, "Characteristics of Synchrotron Radiation," AIP Conf. Proc. $184(565) 1988$

[8] A. H. Lumpkin, L. Emery, and B. X. Yang, "Observations of 'Effective' Transverse Beam Size Instabilities for a High Current per Bunch Fill Pattern in the APS Storage Ring," these proceedings. 